\title{
Modificación de los cólicos nefríticos y de la excreción de calcio en orina de 24 horas en el hiperparatiroidismo primario tras la paratiroidectomía
}

\author{
M.M. Las Heras Alonso, L. Pérez Ruiz, J. Guajardo Guajardo, J.R. Bordalba Gómez, \\ R. Puig Giró, M. Gómez Agudo, L.M. Flavian Domenech, C. Berbegal
}

Departamento de Urología, Hospital Universitario Arnau de Vilanova, Lleida.

Actas Urol Esp 2005: 29 (3): 292-295

\section{RESUMEN}

MODIFICACIÓN DE LOS CÓLICOS NEFRÍTICOS Y DE LA EXCRECIÓN DE CALCIO EN ORINA DE 24 HORAS EN EL HIPERPARATIROIDISMO PRIMARIO TRAS LA PARATIROIDECTOMÍA

Se documentó la frecuencia de cólicos nefríticos en pacientes con hiperparatiroidismo primario, y se determinó su modificación tras la paratiroidectomía; así mismo se estudiaron parámetros de laboratorio tales como calcio, fósforo y parathormona en suero, y excreción de calcio en orina de $24 \mathrm{~h}$ previos y posteriores a la intervención. A la vista de los resultados se puede concluir que la paratiroidectomía es útil en el tratamiento de la enfermedad calculosa renal producida por el hiperparatiroidismo primario.

Palabras clave: Cólico nefrítico. Excreción Cao 24h. Paratiroidectomía.

\section{ABSTRACT \\ MODIFICATION OF NEPHRITIC COLIC AND 24-HOUR CALCIUM EXCRETION IN URINE IN PRIMARY HYPERTHYROIDISM AFTER PARATHYROIDECTOMY}

We documented the frequency of nephritic colic in patients with primary hiperparathyroidism, and determined its modification after the parathyroidectomy; we also studied laboratory parameters such as calcium, phosphorus and parathyroid hormone in serum, and the excretion of Cao $24 \mathrm{~h}$, previous and later to the intervention. At sight of the results it is possible to be concluded that the parathyroidectomy is useful in the treatment of the kidney stone disease produced by the primary hiperparathyroidism.

Keywords: Nephritic colic. Excretion of Cao 24h. Parathyroidectomy.

$\mathrm{E}$ 1 hiperparatiroidismo primario es un trastorno generalizado del metabolismo del calcio, de los fosfatos y del hueso, debido a un aumento de secreción de la hormona paratiroidea. Esta enfermedad afecta a un 5\% de la población con litiasis, y es una causa reversible de nefrolitiasis.

\section{MATERIAL Y MÉTODOS}

Se estudiaron 39 pacientes con hiperparatiroidismo primario sintomático, que fueron tratados mediante paratiroidectomía entre enero de 1992 y diciembre de 2002. Todas las intervenciones fueron realizadas por el mismo cirujano en el Hospital Universitario Arnau de Vilanova de Lleida. Se recogió de la historia clínica de cada uno de los pacientes la información sobre la presencia o ausencia de cólicos nefríticos, y se realizó una encuesta telefónica interrogando a todos los pacientes sobre la presencia o ausencia de ellos tras la intervención, en un periodo postoperatorio comprendido entre los 6 meses y los 11 años. Se obtuvieron los valores de calcio, fósforo, parathormona y calcio en orina de $24 \mathrm{~h}$; se tomó una muestra preoperatoria, una primera muestra 
postoperatoria inmediata y una segunda muestra tras un tiempo de seguimiento que varió de los 2 años a los 5 años tras la intervención quirúrgica.

El propósito de esta investigación fue estudiar:

- La frecuencia de los cólicos nefríticos en pacientes con hiperparatiroidismo primario, determinando si éstas se resuelven tras paratiroidectomía.

- El valor de calcio, fósforo y hormona paratiroidea previo a la intervención, según la presencia o ausencia de cólicos nefríticos.

- La excreción de calcio en orina de $24 \mathrm{~h}$ previa a la intervención según la presencia o ausencia de cólicos nefríticos, así como los valores de dicho parámetro antes y después de la paratiroidectomía, estudiando si se normaliza tras la intervención.

Se utilizaron las pruebas de chi-cuadrado. Anova de 1 factor, y T-student para el análisis de datos.

\section{RESULTADOS}

Se obtuvo una disminución significativa en la frecuencia de aparición de cólicos nefríticos, que pasó a ser del $41 \%$

(16 pacientes) previo a la intervención a 5,1\% ( 2 pacientes) posteriormente a la intervención ( $\mathrm{p}=0,000)$ (Fig. 1).

No se encontraron diferencias significativas en los valores de calcio, y PTH previos a la paratiroidectomía entre los pacientes sintomáticos y los asintomáticos, pero sí en el valor del fósforo, que fue mayor de forma significativa $(\mathrm{p}=0,019)$ en los que no presentaban cólicos $(2,6 \mathrm{mg} / \mathrm{dl})$ respecto de los que sí lo presentaban $(2,3 \mathrm{mg} / \mathrm{dl})$ (Fig. 2).

La media de excreción de calcio en orina de $24 \mathrm{~h}$ de forma previa a la paratiroidectomía es mayor en los pacientes con cólicos $(417,0 \mathrm{mg} / 24 \mathrm{~h})$ que en los que no tienen cólicos (341,6 mg/24h) pero no se halla diferencia significativa (Fig. 3).

Considerando todo el grupo de enfermos, la media de la excreción de $\mathrm{Ca}_{\mathrm{o}} 24 \mathrm{~h}$ es menor en el primer control postoperatorio que previamente a la intervención, pero no de forma significativa, pero en el control de seguimiento, esta excreción es menor $(213,1 \mathrm{mg} / 24)$ y de forma significativa respecto al momento previo a la intervención (406,8 mg/24h) (Fig. 4).

Si se consideran sólo los pacientes sin cólicos previos, la excreción del $\mathrm{Ca}_{\mathrm{o}} 24 \mathrm{~h}$ es menor y de forma significativa en el primer control postoperatorio $(194 \mathrm{mg} / 24 \mathrm{~h})$ que previamente a la intervención. Esta excreción es menor (181 mg/24h) y de forma significativa en el control de seguimiento respecto al momento previo a la intervención (Fig. 4).

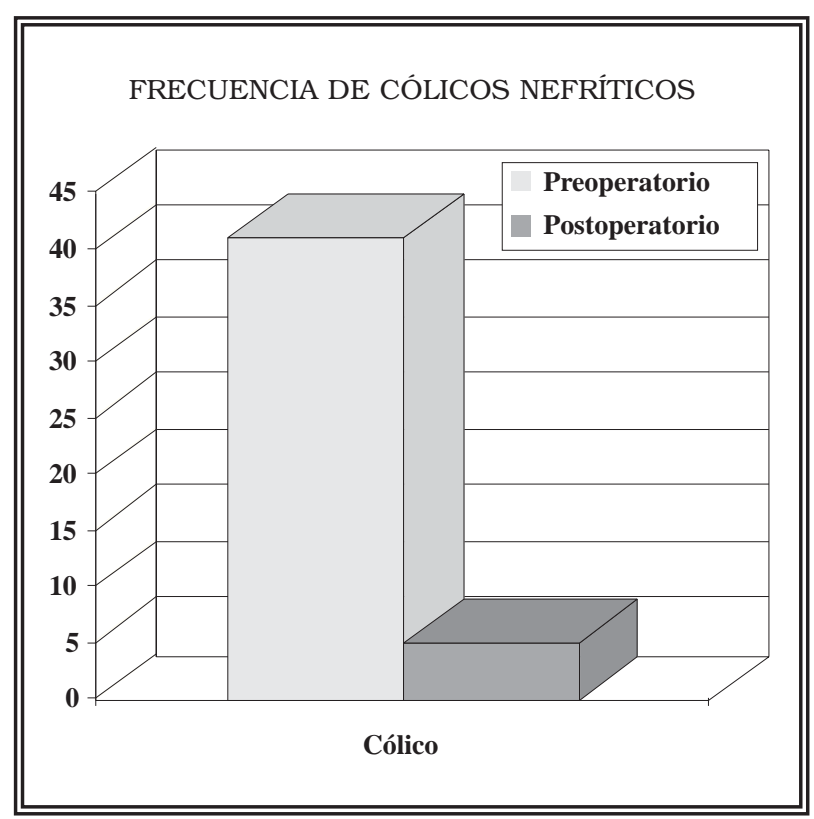

FIGURA 1

\begin{tabular}{|c|c|c|c|c|c|c|}
\hline \multicolumn{7}{|c|}{ Ca, P, PTH PREOPERATORIOS } \\
\hline & $\underline{\mathrm{Ca}}$ & & $\underline{\mathrm{P}}$ & & $\underline{\text { PTH }}$ & \\
\hline & Presencia & Ausencia & Presencia & Ausencia & Presencia & Ausencia \\
\hline $\begin{array}{l}\text { Cólicos } \\
\text { nefriticos }\end{array}$ & 11,6 & 11,2 & & & 14,8 & 18,1 \\
\hline
\end{tabular}

FIGURA 2 


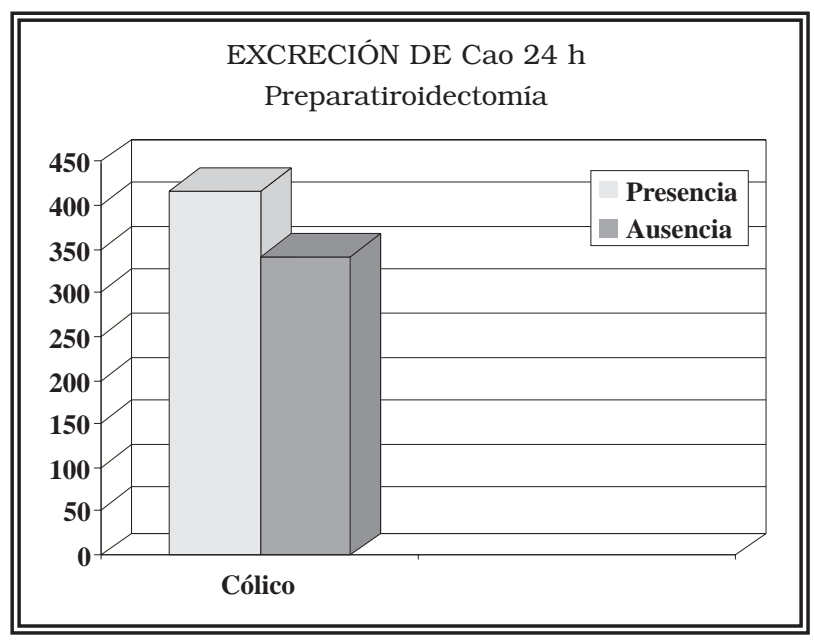

FIGURA 3

En los pacientes con cólicos previos, la excreción del $\mathrm{Ca}_{\mathrm{o}}$ 24h no disminuye de forma significativa en el primer control postoperatorio respecto del momento previo a la intervención, pero sí disminuye y de forma significativa $(\mathrm{p}=0,038)$ en el control de seguimiento $(240$ $\mathrm{mg} / 24 \mathrm{~h}$ ) respecto al momento previo a la intervención (Fig. 4).

\section{DISCUSIÓN}

Hemos constatado, que la paratiroidectomía exitosa (una o más glándulas paratiroideas anormales extirpadas y el nivel de calcio sérico normalizado) frecuentemente resulta en la resolución o mejora de los cólicos nefríticos en los pacientes con hiperparatiroidismo.

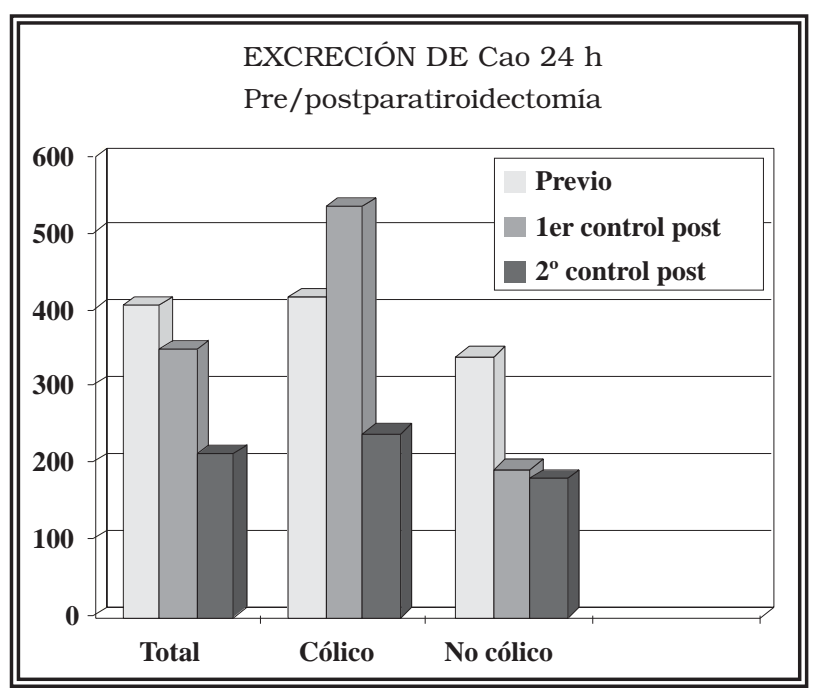

FIGURA 4
En lo que se refiere a la excreción de calcio en orina de 24 horas, comparando los pacientes con cólico nefrítico y los que no lo presentan, al igual que V.G. Frokjaer et al. ${ }^{1}$, no encontramos diferencias significativas en las determinaciones preoperatorios de dicho parámetro, pero sí se produce una reducción significativa tras la intervención.

La formación de cálculos de calcio entre los pacientes con hiperparatiroidismo primario ha sido atribuida a la hipercalciuria, aunque otros factores promotores de cálculos y la disfunción tubular han sido debatidos. Se ha demostrado que la excreción de calcio urinario se reduce después de la paratiroidectomía exitosa, y la enfermedad calculosa renal asociada con hiperparatiroidismo primario es considerada curada tras la correción de la hipercalcemia, aunque esto ha sido cuestionado por los anteriores autores, que comparando los pacientes con y sin enfermedad calculosa renal, vieron que los formadores de cálculos mostraban una mayor excreción de calcio postoperatoriamente, lo que podría indicar que otros factores diferentes al hiperparatiroidismo, tales como la hipercalciuria idiopática, podrian jugar un papel en estos pacientes. El exceso de calcio en la orina puede proceder de un aumento de la absorción de calcio en el intestino, de un pérdida renal o de un aumento de la movilización del calcio de los huesos. Una posibilidad es que la hipercalciuria idiopática puede, en algunos casos, ser la causa de un posterior hiperparatiroidismo primario $^{3}$. Esto explicaría por qué la enfermedad calculosa renal ocurre muchos años antes del diagnóstico, y concuerda con el incremento persistente en el riesgo de litiasis renal varios años tras la cirugía de paratiroides, especialmente entre pacientes que previamente tuvieron cálculos. Esta interpretación va a favor de que la pérdida de calcio continúa tras la paratiroidectomía, y concuerda con nuestros hallazgos de una mayor excreción de calcio en orina de $24 \mathrm{~h}$ en la primera muestra postoperatoria en los pacientes con cólicos nefríticos respecto a los que no los presentaban. Johansson ${ }^{5}$ sugiere que el diagnóstico de hipercalciuria idiopática no debe ser hecho a menos que las glandulas paratiroides hayan sido exploradas, porque en su experiencia muchos pacientes diagnosticados de hipercalciuria idiopática padecían hiperparatiroidismo primario. La pérdida de calcio en orina conduciría a un hiperparatiroidismo secundario, por eso la cirugía en 
estos pacientes no conseguiría buenos resultados. En estos pacientes se debería intentar reducir la excreción de calcio urinario con tiazidas, en el caso de que la pérdida fuese renal, o con fosfato de celulosa, en el caso de un aumento de la absorción intestinal.

Mollerup refiere que los pacientes con litiasis tienen un mayor riesgo de estenosis de ureter y de hidronefrosis que los controles. Esto puede indicar que los cálculos causan un daño anatómico al tracto urinario, y estos cambios estructurales podrían incrementar el riesgo de padecer cálculos tras la cirugía, incluso si las anormalidades bioquímicas han sido normalizadas. La enfermedad calculosa renal es una enfermedad multifactorial, lo que puede explicar por qué algunos pacientes con hiperparatiroidismo primario desarrollan nefrolitiasis y otros no. Según Mollerup ${ }^{2}$, el porcentaje de recurrencia de los cálculos a los 5 años de la paratiroidectomía es del $30 \%$, porcentaje comparable al de los pacientes con enfermedad calculosa idiopática. Este autor defiende que la enfermedad calculosa renal depende de varios factores de riesgo, y que la reducción de factores tales como la excreción de fosfato, el calcio en orina y el pH urinario tras la paratiroidectomía podrían tener un efecto beneficioso en la recurrencia de cálculos renales.

Al igual que Söreide et al. ${ }^{4}$, nosotros no hemos encontrado diferencias en los valores de calcio y parathormona entre los pacientes con cólicos nefríticos y sin ellos. Ellos creen que desde que el diagnóstico de hiperparatiroidismo es más fácilmente realizado gracias a los análisis bioquímicos, la severidad de la hipercalcemia ha cambiado drásticamente. Esto puede explicar en parte por qué los pacientes con cólicos nefríticos en nuestro estudio no muestran un patrón bioquímico diferente comparado con los pacientes sin cólicos nefriticos.

Otra teoría contempla un posible efecto de la parathormona en el metabolismo del oxalato durante los estadíos iniciales de la nefrolitiasis; Yamaguchi et al. ${ }^{6}$ detectaron fosfato cálcico y oxalato cálcico en las placas incipientes de litiasis en los túbulos renales, localizados principalmente en la unión corticomedular. El aumento del número de placas estuvo asociado con un nivel mayor de hormona paratiroide.

\section{CONCLUSIÓN}

La indicación de paratiroidectomía está en función de los sintomas clínicos y desórdenes metabólicos, de su severidad y de su reversibilidad tras la cirugía. La información obtenida apoya a la paratiroidectomía como tratamiento para los pacientes con hiperparatiroidismo primario y cólicos nefríticos.

\section{REFERENCIAS}

1. Frokjaer VG, Mollerup CL. Primary hyperparathyroidism: renal calcium excretion in patients with and without renal stone disease befote and alter parathyroidectomy. World J. Surg 2002;26:532-535.

2. Mollerup CL, Lindewald H. Renal stones and primary hyperparathyroidism: natural history of renal stone disease after successful parathyroidectomy. World J. Surg1999;23:173-176.

3. Mollerup CL, Vestergaard P, Frokjaer VG, Mosekilde L, Christiansen P, Blichert-Toft M. Risk of renal stone events in primary hyperparathyroidism before and after parathyroid surgery: controlled retrospective follow up study. BMJ 2002:October 12;325(7368):807.

4. Söreide JA, Van Heerden JA, Grant CS, Lo CY, Ilstrup DM. Characteristics of patients surgically treated for primary hyperparathyroidism with and without renal stones. Surgery 1996;120(6):1033-1038.

5. Johansson H, Thorén L., Werner I, Grimelius L. Normocalcemic Hyperparathyroidism, kidney stones, and idiopathic hypercalciuria. Surgery 1975;77(5):691-696

6. Yamaguchi S, Yachiku S, Okuyama M, Tokumitsu M, Kaneko S, Tsurukawa.H. Early stage of urolithiasis formation in experimental hyperparathyroidism. The Journal of Urology 2001;165:1268-1273.

7. Van Heerden JA, Segura JW, Grant CS. Primary hyperparathyroidism and urolithiasis: concomitant surgical management. Surgery 1989; 106(6):992-996.

8. Evans RA, Hills EL, Wong S, Wyndham LE, Eade Y, Dunstan CR. The pathogenesis of idiopathic hypercalciuria: evidence for parathyroid hyperfunction. Quarterly Journal of Medicine 1984,209:41-53.

9. Deaconson TF, Wilson SD, Lemann J. The effect of parathyroidectomy on the recurrence of nephrolithiasis. Surgery 1987;102(6):910-913.

10. De Madrid DP, Muñoz M, Escobar F, Rodríguez C, Zuloaga A, Morales I. Perfil bioquímico del hiperpartiroidismo primario. Estudio comparativo con la litiasis renal hipercalciúrica. Medicina Clinica 1989;92 (13):481-483.

11. Pejenaute MC, Llerena R, Pertusa C, Vázquez JA. Manifestaciones urológicas del hiperparatiroidismo primario. Arch. Esp. de Urol 1991; 44(4):425-428

12. Wells SA. Surgical Therapy of patients with primary hyperparathyroidism: long-term benefits. Journal of Bone and Mineral Research. 1991;6(2):143-149

13. Parks J, Coe F, Favus M. Hyperparathyroidism in nephrolitiasis. Arch Intern Med 1980,140:1479-1481.

14. Dimkovic NB, Wallele AA, Oreopoulos DG. Renal stone disease, elevated I PTH level and normocalcemia. International Urology and Nephrology 2002;34:135-141.

15. Chan AK, Duh Q, Katz M, Siperstein AE, Clark OH. Clinical manifestations of primary hyperparathyroidism before and after parathyroidectomy. Annals of Surgery 1995; 22(3):402-414.

16. Pak C. Should patients with single renal stone occurrence undergo diagnostic evaluation?. The Journal of Urology 1982;127:855-858.

Dra. M.M. Las Heras

Hospital Univ. Arnau de Vilanova

Avda. Alcalde Rovira Roure, 80

25198 Lleida

(Trabajo recibido el 11 noviembre de 2004) 